\title{
Granizada e inundación del 19 de febrero de 2002. Un modelo de crisis para la aglomeración de La Paz
}

Orage de grêle et inondation du 19 février 2002. Un modèle de crise pour

l'agglomération de La Paz

Hail storm and flood of February, 19th, 2002. A crisis model for the

agglomeration of La Paz

\section{Sébastien Hardy}

\section{OpenEdition \\ Journals}

Edición electrónica

URL: http://journals.openedition.org/bifea/2300

DOI: $10.4000 /$ bifea.2300

ISSN: 2076-5827

Editor

Institut Français d'Études Andines

Edición impresa

Fecha de publicación: 1 diciembre 2009

Paginación: 501-514

ISSN: 0303-7495

Referencia electrónica

Sébastien Hardy, «Granizada e inundación del 19 de febrero de 2002. Un modelo de crisis para la aglomeración de La Paz », Bulletin de l'Institut français d'études andines [En línea], 38 (3) | 2009,

Publicado el 01 junio 2010, consultado el 17 noviembre 2020. URL : http://journals.openedition.org/ bifea/2300 ; DOI : https://doi.org/10.4000/bifea.2300

\section{BY NE ND}

Les contenus du Bulletin de l'Institut français d'études andines sont mis à disposition selon les termes de la licence Creative Commons Attribution - Pas d'Utilisation Commerciale - Pas de Modification 4.0 International. 


\title{
Granizada e inundación del 19 de febrero de 2002. Un modelo de crisis para la aglomeración de La Paz
}

\author{
Sébastien Hardy*
}

\section{Resumen}

El 19 de febrero de 2002, una granizada azotó la ciudad de La Paz. Causó innumerables daños tanto a los individuos como a las infraestructuras, por lo que fue calificada de crisis. El evento se presentó como un desastre natural excepcional. Sin embargo, el análisis retrospectivo de este evento muestra que la crisis se desató a causa de una vulnerabilidad estructural cuyos factores no hicieron sino entrar en resonancia los unos con los otros, el 19 de febrero. Los gestores de crisis sacaron algunas lecciones de este evento. Empero, las decisiones asumidas no han reducido aún la vulnerabilidad estructural.

Palabras clave: riesgo, vulnerabilidad, elementos esenciales, temporalidad, La Paz

\section{Orage de grêle et inondation du 19 février 2002. Un modèle de crise pour l'agglomération de La Paz}

\section{Résumé}

Le 19 février 2002 un orage de grêle s'est abattu sur la ville de La Paz. Il a provoqué de tels dommages, à la fois aux individus et aux infrastructures, qu'il a été qualifié de crise. L'évènement a ainsi été présenté comme une catastrophe naturelle exceptionnelle. Pourtant, son analyse en retour montre que la crise a largement été préparée par une vulnérabilité structurelle dont les facteurs n'ont fait qu'entrer en résonnance les uns par rapport aux autres le 19 février. Les gestionnaires de crises ont tiré certaines leçons de cet évènement, sans pour autant que les décisions aient véritablement réduit la vulnérabilité structurelle.

Mots clés : risque, vulnérabilité, enjeu, temporalité, La Paz

* Institut de Recherche pour le Développement (IRD), UR 029, programa Pacivur, Av. Hernando Siles 5290 esq. calle 7, CP 9214 Obrajes, La Paz, Bolivia. E-mail: sebastien.hardy@ird.fr 


\title{
Hail storm and flood of February, 19th, 2002. A crisis model for the agglomeration of La Paz
}

\begin{abstract}
On February 19, 2002 a hail storm came down on the city of La Paz. It caused such damage, both to individuals and infrastructure, that it was described as a crisis. The event was thus presented as an exceptional natural disaster. However, in retrospective, the analysis shows that the crisis was largely caused by a structural vulnerability, whose factors interacted with one another. Crisis managers learned certain lessons from this event; noneheles, their decisions didn't truly reduce structural vulnerability.
\end{abstract}

Key words: risk, vulnerability, strategic elements, stages, La Paz

\section{DESCRIPCIÓN Y CONTEXTO}

El 19 de febrero de 2002, entre las 14:20 y las 15:45, una granizada azotó la parte central de la ciudad de La Paz. Es un fenómeno meteorológico habitual en época de lluvias (diciembre-marzo). En efecto, el ascenso de un aire algo más húmedo que durante el resto del año, es favorecido por las temperaturas medias más cálidas del verano andino. No obstante, puede ser muy brutal, forzado localmente por la topografía de la hondonada con declives abruptos (BCEOM, 1977; Demoraes, 1998) donde la aglomeración de La Paz se despliega entre 3200 y 4100 metros de altura. El aire, brutalmente propulsado en altura, se satura muy rápidamente al contacto con el aire más frío que encuentra al elevarse. Su humedad se transforma entonces en hielo desencadenando granizadas localizadas sobre la aglomeración paceña.

Aquel 19 de febrero, la masa de nubes sobre La Paz presentó una altura de 10 kilómetros y alimentó una granizada que duró una hora y media, tiempo durante el cual la temperatura media en el suelo pasó de 13 a 8 grados Celsius (Villegas, 2002: 19). Ese día, el volumen total de las precipitaciones registradas alcanzó una altura de $41 \mathrm{~mm}$ de los cuales $39,4 \mathrm{~mm}$ en apenas una hora. Es el evento más fuerte registrado desde 1976, fecha en la cual el volumen había alcanzado $32 \mathrm{~mm}$ (OPS, 2002).

Muchos testimonios describen la brutalidad del aguacero. Por ejemplo, Yobanca Fernández, presidenta del barrio Palermo, recuerda:

«Estaba en El Alto, mi auto estaba averiado así que fui a buscar a mi hermano que es mecánico. Comenzó a llover, y cuando levanté los ojos hacia el cielo, había una enorme nube negra que avanzaba sobre La Paz. No comprendimos inmediatamente. Llovía muchísimo, las calles de El Alto estaban inundadas y ni siquiera podíamos imaginarnos lo que pasaba en La Paz» (entrevista del 01/02/07 con Yobanca Fernández) (Combaz, 2007). 


\section{CONSECUENCIAS}

La granizada provocó muchas inundaciones y deslizamientos de tierra en la aglomeración paceña, ocasionando a su vez importantes estragos. Los recuentos varían de un organismo a otro, pero se puede enumerar al menos 69 muertos - la mayor parte fueron vendedoras de la calle del sector informal - 130 heridos y 50 desaparecidos. Los daños estructurales fueron evaluados en 10 millones de dólares (daños en las vías de comunicación, los vehículos, los edificios públicos y privados como el centro de salud Policonsultorio de la Caja Nacional de Salud en la Avenida Manco Kapac), además de las interrupciones de alimentación en energía eléctrica y en agua potable (Villegas, 2002; OPS, 2002; GMLP, 2007; Nathan, 2008: 340) que agravaron los disfuncionamientos de la fase de urgencia. Además, aproximadamente 200 familias se vieron obligadas a abandonar sus viviendas damnificadas (Combaz, 2007).

Al cartografiar los espacios urbanos afectados (fig. 1) a partir de los casos de urgencia atendidos por los servicios del Gobierno Municipal de La Paz (GMLP) el 19 de febrero de 2002, se constata que la granizada no afectó de manera homogénea el territorio de la aglomeración de La Paz. La distribución de las intervenciones de urgencia a nivel de los macrodistritos del municipio de La Paz determinó tres espacios: el centro, la parte noroeste y la parte sur de la ciudad.

Esta distribución espacial se explica primero por el proceso meteorológico: la granizada se concentró en el centro y la parte noroeste de la ciudad, antes que las aguas de escorrentía pluvial confluyeran en el fondo de la hondonada, es decir en la parte sur de la ciudad.

Por otro lado, aunque su superficie sea reducida en relación a los otros macrodistritos de la ciudad, el centro ha tenido el mayor número de intervenciones de urgencia, lo que da una idea de los problemas que ha encontrado. Por cierto, el registro de las intervenciones de urgencia del GMLP sólo traduce el número de constataciones de daños, y no la evaluación de los daños. Así el macrodistrito San Antonio no muestra un número elevado de intervenciones de urgencia, cuando los daños sufridos fueron evaluados como mayores a los del macrodistrito Max Paredes, donde las intervenciones fueron más numerosas.

A pesar de esto, la observación de la distribución de las intervenciones de urgencia en La Paz permite hacerse una idea de los espacios afectados y, a partir de ahí, de la crisis provocada por su alteración. El análisis posterior de la granizada permite abordar así los factores estructurales recurrentes de vulnerabilidad que favorecen una mejor comprensión de las posibles crisis.

\section{FACTORES DE VULNERABILIDAD}

Los factores estructurales de vulnerabilidad identificables en La Paz son de varios tipos, generalmente relacionados, más o menos estrechamente, unos con otros. Por ende, el conocimiento preciso de los fenómenos físicos, principalmente de 


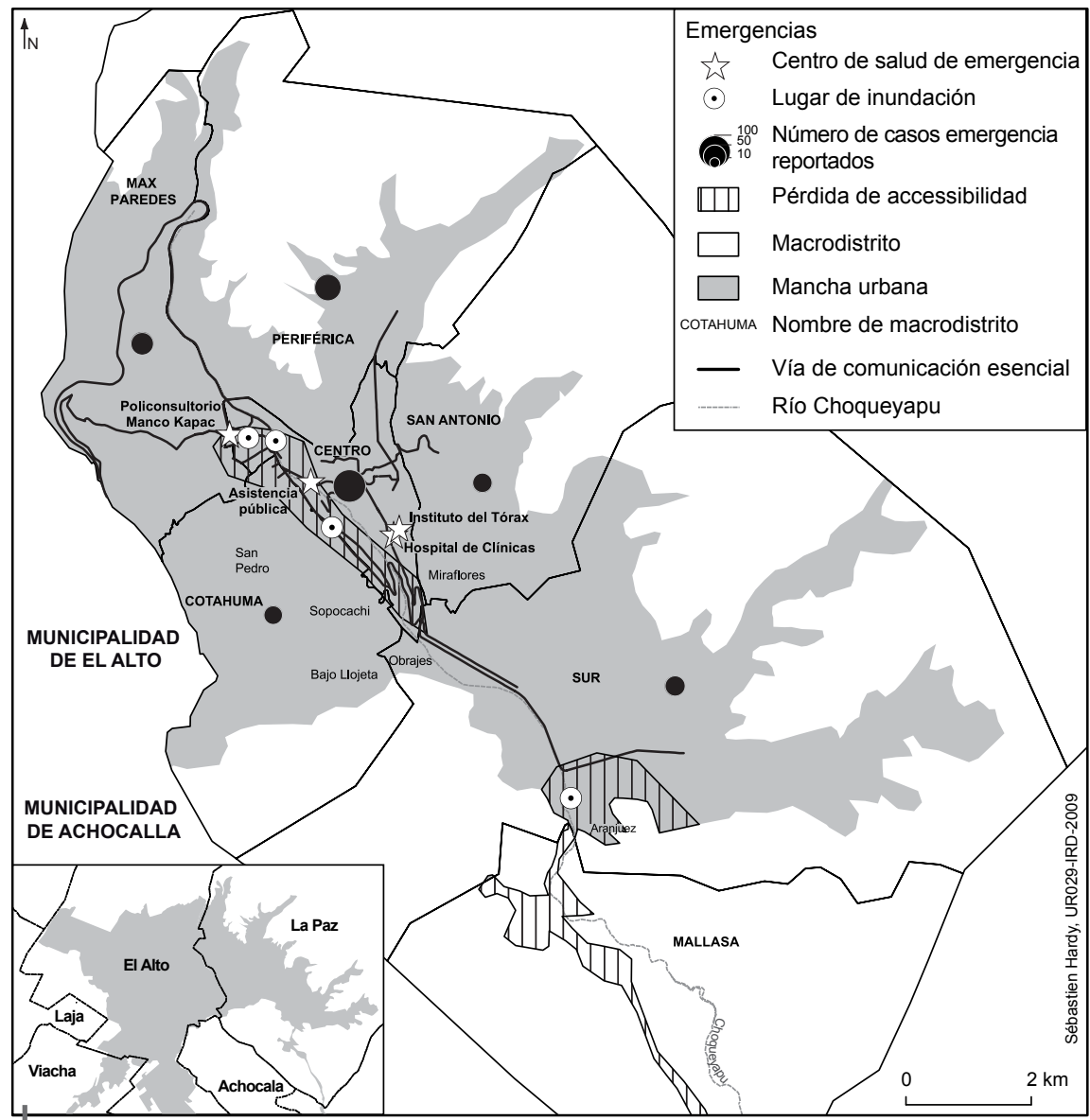

Figura 1 - La distribución de las urgencias en La Paz en respuesta a la granizada y la inundación del 19 de febrero de 2002

su ocurrencia, constituye un primer paso para prever los problemas que puedan desencadenarse. En Bolivia, el Servicio Nacional de Meteorología e Hidrología (SENAMHI) es responsable de la previsión meteorológica. No obstante, este ya no dispone de una red de estaciones meteo-hidrológicas muy densa. En 1980, administraba unas 1800 estaciones. Debido a una disminución del presupuesto, ligada a las crisis económicas de los años 1980 y a los ajustes estructurales, el SENAMHI no administraba sino 353 en el 2007. Pero estas estaciones no proporcionan en tiempo real la información que registran $y$, por otro lado, el SENAMHI no tiene radar para monitorear el desplazamiento rápido de las masas de aire localizadas (Villegas, 2002: 19). En estas condiciones, le es difícil establecer previsiones referentes a las granizadas, principalmente en términos de ubicación, y transmitir la información a los actores implicados en la gestión de los riesgos. Esta deficiencia de información es un primer factor estructural de vulnerabilidad de la aglomeración de La Paz, que traduce la propia vulnerabilidad de las instituciones. 
Los diferentes organismos y actores que pueden intervenir en la gestión de los riesgos son potencialmente numerosos (GMLP, Defensa civil boliviana, Servicio departamental de salud, etc.). Sin embargo, intercambian muy poca información entre sí. Además, en caso de desastre, no se ha previsto procedimiento alguno para coordinar sus acciones en el tiempo y en el espacio, en menosprecio del buen desarrollo de las urgencias y auxilios. Sin embargo, en Bolivia existe un marco legal que rige la gestión de los riesgos que principalmente define los roles de los diferentes actores (Hardy, 2009). Pero esta repartición precisa de las tareas de cada uno y la necesaria coordinación de los actores se concretan con dificultad. En consecuencia, las políticas de prevención se limitan esencialmente a construcciones de obras de ingeniería destinadas a resistir a la manifestación de las amenazas. Estos desórdenes de tipo institucional en la ciudad sede del gobierno boliviano son sorprendentes considerando que el espacio es propicio a sufrir daños, por su topografía, por las características geológicas de los terrenos y por los acondicionamientos.

Topografía, geología y acondicionamientos remiten respectivamente a las amenazas y a las condiciones que han conducido a exponerse a estas. De tal manera que la topografía abrupta favorece la rápida concentración de las aguas de escorrentía pluvial que liberan una poderosa energía. Por otra parte, la escorrentía se lleva a cabo en tierras blandas, fácilmente saturables de agua. Estas condiciones facilitan, en caso de precipitaciones voluminosas, inundaciones casi simultáneas en diversos espacios de la ciudad, así como deslizamientos y derrumbes de tierras. Pero es también el proceso de urbanización el que conduce en gran medida a la exposición a las amenazas.

La urbanización progresa en forma de glacis, es decir amoldándose a la topografía del valle, a la vez hacia las líneas de cumbre y hacia las líneas de fondo (Baby, 1995; Demoraes, 1998; Hardy, 2009). Este proceso relega a la población más pobre hacia los terrenos más abruptos, los menos estables y menos equipados, sobre las laderas del valle formado por el torrente Choqueyapu. No obstante, las poblaciones más acomodadas no están forzosamente más favorecidas. Ciertamente ocupan terrenos cuya topografía es menos abrupta y son más estables, pero que están situados sobre terrazas construidas en el lecho mayor de muchos torrentes que drenan el valle. Por lo tanto, aquí se combinan la exposición a las amenazas de origen natural y las condiciones de urbanización que las exponen, lo que explica la vulnerabilidad de las formas de urbanización.

Para comprender todas las dimensiones de la vulnerabilidad de las formas de urbanización en La Paz, resulta práctico presentarlas desde arriba hacia abajo del valle. Así, las aguas pluviales, después de haberse escurrido y eventualmente dañado los terrenos de las laderas poco estables del valle, se concentran enseguida en el torrente Choqueyapu: es un canal cubierto que recorre el centro, y en el cual se descargan las aguas del sistema de evacuación pluvial. Este sistema técnico de evacuación de las aguas pluviales, que sólo cubre una pequeña superficie de la ciudad, esencialmente el centro, puede ser fácilmente saturado, sobre todo si a menudo va cargado de aguas residuales. En efecto técnicamente, casi nunca se 
distinguen las dos redes en La Paz. Pero la red de evacuación de las aguas pluviales tampoco ha sido calibrada para soportar las dos funciones: la función de evacuación de las aguas residuales resulta, en la mayoría de los casos, de conexiones ilegales en las bocas de desagüe del sistema pluvial. Además, las tormentas de granizo provocan fácilmente la obstrucción de alcantarillas, obligando así a las aguas de escorrentía a concentrarse en las calles y provocando daños. Aquí, el aspecto técnico de ingeniería urbana juega su parte en la vulnerabilidad estructural.

Un poco más abajo, hacia el fondo del valle, a la altura del barrio Obrajes, el torrente Choqueyapu ya no está canalizado, mientras que el declive de su perfil a lo largo se estabiliza, para volverse horizontal. Las aguas drenadas en el centro llegan entonces violentamente a Obrajes, casi bajo presión a causa del efecto de desagüe de las obras de ingeniería hidráulica en el centro, en un lugar donde el bajo declive del torrente disminuye la evacuación de las aguas. En esta parte de la ciudad, el torrente entra fácilmente en crecida inundando espacios donde las infraestructuras son numerosas (avenida Costanera, Hospital de la Caja Petrolera, etc.) y muy expuestas al daño. En efecto, estos barrios de la parte sur de la ciudad han sido edificados en el lecho mayor del torrente Choqueyapu y en el lecho mayor de sus afluentes. Esta elección de localización se justificó con la realización de obras de ingeniería hidráulica de protección, a pesar de la cartografía de los terrenos construibles realizada en 1977 (BCEOM, BRGM y PCA, 1977) que identifica estas zonas como inconstruibles por su exposición a la inundación. De arriba hacia abajo del valle de La Paz, las obras de acondicionamiento (canalizaciones, gaviones, etc.) así como las modalidades de ocupación de los terrenos, favorecen por lo tanto el daño, tanto como los fenómenos físicos, y además están ligadas a éstos. Tanto uno como otro aparecen como factores estructurales de la vulnerabilidad de la ciudad de La Paz.

Otras vulnerabilidades estructurales se desprenden de esta descripción nortesur de la ciudad, aquellas que están relacionadas con la presencia de elementos esenciales (D’Ercole \& Metzger, 2004) en estos diferentes espacios. El macrodistrito Centro es el principal espacio de actividades de la aglomeración. Reúne empleos formales (administraciones del Estado, del departamento y de la municipalidad; sedes del Banco Central y de los principales bancos bolivianos, etc.) y empleos informales (puestos de venta de alimentos y bebidas, lustrabotas, etc.). Por sus características, este espacio genera flujos intensos que favorecen la convergencia de la población. Por lo tanto, concentra elementos esenciales importantes cuya pérdida podría provocar graves dificultades: por ejemplo, la paralización de las actividades bancarias en el país, de las decisiones políticas y de gestión, etc. Además, el análisis de los elementos esenciales concentrados en el centro muestra que son en sí mismos bastante vulnerables. En el caso de los empleos informales, consisten en puestos de venta en la calle, con una infraestructura frágil, donde los trabajadores están directamente expuestos a las amenazas. Estos puestos por lo general son atendidos por mujeres, de origen indígena, que en su mayoría llevan los vestidos tradicionales, a saber, una superposición de faldas hechas de tela gruesa. El factor cultural relacionado a la vestimenta forma parte también de la vulnerabilidad de la población del sector informal del centro de la ciudad que los 
hace menos móviles en caso de urgencia. En fin, la accesibilidad a este espacio que concentra elementos esenciales, por ejemplo para los medios de auxilio, resulta igualmente difícil a causa de la red vial, que toma una forma muy particular en esta parte de la ciudad. La red vial de la aglomeración paceña es longitudinal, orientada de norte a sur a causa de la topografía que le deja poco espacio para desplegarse de este a oeste. Igualmente a causa de su topografía, su densidad puede ser fuerte en ciertas zonas de la ciudad, como en el caso del centro donde se superpone a los lechos drenados de los torrentes. La pérdida de las vías de comunicación en el centro significa por lo tanto su pérdida de accesibilidad desde y hacia las otras partes de la ciudad, mostrando así que las vías de comunicación constituyen un elemento de la vulnerabilidad estructural del centro. Se puede constatar la vulnerabilidad de accesibilidad en muchas otras partes de la ciudad.

Todos estos factores de vulnerabilidad estructural han actuado juntos y separadamente para desencadenar la crisis del 19 de febrero de 2002.

\section{GESTIÓN DE CRISIS}

Estos factores de la vulnerabilidad estructural de La Paz tuvieron incidencias sobre la gestión de la granizada, transformando este evento en una crisis. Así, la vulnerabilidad institucional antes mencionada explica la inexistencia, en febrero de 2002, de un procedimiento de transmisión de los boletines meteorológicos del SENAMHI a las instituciones encargadas de afrontar una eventual crisis, como el GMLP o la Defensa Civil boliviana, para que ellas puedan anticipar una violenta granizada, tomando por ejemplo disposiciones adecuadas. Aún cuando actualmente el SENAMHI sólo es capaz de proporcionar previsiones meteorológicas de orden muy general, y no alertas, la ausencia de transmisión sistemática de información el 19 de febrero no permitió a los gestores de crisis estar listos para intervenir.

De todas maneras, a pesar de los eventos anteriores (granizada de enero de 1973, de enero de 1976, de enero de 1982, de abril de 1988, etc.), cuando ocurrió la granizada del 19 de febrero, el GMLP no tenía una organización prevista para enfrentarse a una crisis: responsabilidades definidas de los actores, cadena de decisiones, procedimientos preestablecidos (Hardy, 2009). La misma constatación vale para los otros actores potenciales de gestión de crisis, como la Defensa Civil boliviana. La gestión de la urgencia se operó entonces desordenadamente, tanto así que muchos actores intervinieron simultáneamente: personal de diferentes direcciones del GMLP, policías de la Patrulla 1102, bomberos, voluntarios del grupo SAR (Search and rescue)3, militares de las Fuerzas armadas bolivianas, personal de urgencia del Servicio departamental de salud (SEDES La Paz), etc.

1 Ver en este número del Bulletin de I'IFEA, el artículo de Nuñez \& Demoraes, pp. 827-848.

2 La policía de la Patrulla 110 es uno de los cuerpos de la Policía Nacional boliviana, específicamente encargada de la prevención y ayuda a los ciudadanos.

3 Fundado en Bolivia en 1992, el grupo SAR (Search and rescue), que funciona bajo el régimen legal de una organización no gubernamental, está compuesto de voluntarios que reciben capacitaciones 
Durante la fase de la urgencia, en ausencia de plan de auxilio predefinido, sus acciones no fueron coordinadas, ni en el tiempo ni en el espacio, en detrimento de la mejor operatividad esperada de los auxilios de urgencia. Por cierto, espontáneamente se formaron equipos de auxilio de urgencia. Algunos se ocuparon de despejar los granizos de las vías de comunicación; otros de buscar víctimas y evacuarlas para darles asistencia; algunos de restablecer la electricidad, o de reparar las canalizaciones de drenaje pluvial, etc. Pero generalmente estos equipos se formaron sin tener en cuenta las especializaciones de cada uno de sus miembros y, por lo tanto, de su mejor utilidad. Asimismo, el despeje de las vías de circulación se operó rápidamente pero sin estrategia de intervención, la cual podría haberse basado en una jerarquía de vías a despejar en prioridad, establecida en función de su importancia para conectar el espacio afectado y en crisis con los espacios que podrían haber brindado acceso a los primeros auxilios: centros de salud, bomberos, etc.

En medio de este desorden, el ministerio de Salud pudo desbloquear rápidamente 30000 \$ US para aportar los primeros auxilios a las víctimas en tres centros de salud movilizados con esta ocasión (Hospital de Clínicas, Hospital del Tórax, Asistencia Pública). Por otro lado el personal de salud, que estaba en huelga de hambre protestando contra sus lamentables condiciones de trabajo, suspendió su acción para asumir inmediatamente sus responsabilidades frente a la crisis y atender a las víctimas.

Durante los días que siguieron a la tormenta, los roles de cada uno se aclararon muy rápidamente y la organización se volvió más eficaz. En primer lugar, frente a la magnitud de los daños, el 20 de febrero, Jorge Quiroga, Presidente de la República de Bolivia, emitió el decreto supremo n. ${ }^{\circ} 26504$, que declaró La Paz y sus alrededores «zona de catástrofe nacional». Esta declaración permitió poner los medios del Estado al servicio de la resolución de la urgencia en el municipio de La Paz. El Presidente de la República pudo entonces asignar 10 millones de dólares para la urgencia y los auxilios a los damnificados (Villegas, 2002: 23). También pidió a la Prefectura del departamento de La Paz canalizar las donaciones espontáneas que las poblaciones hacían a las víctimas. Un acuerdo entre la Conferencia Episcopal Boliviana (CEB), la Prefectura del departamento de La Paz, el GMLP y la Presidencia de la República de Bolivia permitió establecer que la ONG Caritas de la Iglesia Católica romana fuera responsable de la distribución de los alimentos, ropas y medicamentos a los damnificados. La Prefectura pidió a los medios de comunicación solicitar donaciones y habilitó una línea telefónica gratuita para recibirlas. La Asociación Bancaria (ASOBAN) abrió cuentas para administrar las donaciones monetarias. La Prefectura del departamento de La Paz también decidió poner a disposición de las autoridades encargadas las instalaciones deportivas de las cuales es responsable, a fin de albergar a los damnificados y permitirles acceder con mayor facilidad a las donaciones. El viceministerio de Asuntos Sociales instaló células de evaluación de los daños para las víctimas.

específicas para dar auxilio a la población en situación de crisis, principalmente en términos de rescate. 
Por su lado, el ministerio de Educación, a través del Servicio Departamental de Educación de La Paz (SEDUCA) suspendió las actividades escolares, por lo menos hasta el 21 de febrero, a fin de que los damnificados se recuperen y se alberguen en las escuelas, hasta volver a la normalidad. Las autoridades encargadas de la crisis delegaron al Programa de las Naciones Unidas para el Desarrollo (PNUD) la gestión de las donaciones de los gobiernos, de los organismos internacionales y de las empresas. Durante los días que siguieron la granizada, organizaciones vecinales, centrales sindicales, empresas privadas, centrales sindicales, aportaron su apoyo en servicios (transportes, telecomunicaciones y preparación de comidas), y con donaciones monetarias y diversos materiales. Progresivamente, los roles de cada uno de los principales actores de la gestión de la crisis se definieron. Por ejemplo, la Presidencia de la República de Bolivia se encargó de la indemnización de las familias por los miembros fallecidos; la Oficialía de desarrollo económico del GMLP se encargó de indemnizar a los comerciantes y, en coordinación con el ministerio de Vivienda, de reubicar a los damnificados4.

Para comprender por qué esta granizada se transformó en crisis, conviene examinar sus temporalidades (Lagadec, 2002: 164), y vincular las temporalidades a los espacios de la crisis, en todas sus dimensiones. Efectivamente, se tiene el evento en sí, la tormenta, que provoca una crisis inmediata, en espacios dados, expuestos, poco accesibles, donde se concentran poblaciones y actividades, algunas particularmente vulnerables. Luego están las consecuencias del evento -el tiempo del después- que se prolongaron durante semanas después del evento, en espacios dados a menudo diferentes de los primeros. También está el ante-evento. Abordar esas tres dimensiones temporales, enriquecedoras en enseñanzas, permite entender por qué la granizada del 19 de febrero produjo una crisis.

Durante las semanas anteriores al evento se registraron en la aglomeración paceña voluminosas precipitaciones pluviosas que, a veces, se convirtieron en granizadas. Estas contribuyeron en gran medida a preparar la crisis a largo plazo, conduciendo los terrenos a su punto de saturación de absorción de las aguas de escorrentía, facilitando su deslizamiento. De igual manera, hicieron que los ríos, cuyos caudales ya estaban bastante elevados, entraran en crecida súbita el 19.

La temporalidad de la ocurrencia del evento nos conduce a expresar algunos comentarios. La granizada se produjo en plena tarde, un día de semana. La temporalidad del evento, unida al espacio — el centro de La Paz- explica la gran cantidad de damnificados. Las principales víctimas son vendedoras de las calles del sector informal, que trabajaban cuando fueron sorprendidas por la tormenta. La acumulación rápida de granizos en el suelo, la tarde del 19 de febrero, les hizo perder el equilibrio y les dificultó levantarse de un suelo tapizado de granizos. Mientras se debatían en el suelo para ponerse de pie, rápidamente sus faldas se llenaron de agua fría y su peso hizo que les resultara muy difícil enderezarse.

4 Ver en este volumen, el artículo de Hardy \& Combaz, pp. 799-823. 
El factor temporal vinculado al factor espacial, socioeconómico y cultural, explican por qué hubo víctimas, sobre todo mujeres, y por qué murieron de hipotermia.

Por último, la temporalidad de la ocurrencia del evento tuvo repercusiones sobre la gestión de la urgencia. Esta implica que las familias estuvieran a menudo disgregadas: los padres se encontraban en su lugar de actividad profesional, los niños en su lugar de estudios o de distracciones. Las consecuencias de esta temporalidad se encuentran así en los primeros refugios de auxilio: durante la fase de la gestión de la urgencia, las autoridades debieron proceder a la búsqueda de los miembros de familias separadas, principalmente censando a los damnificados.

La temporalidad vinculada al espacio afectado explica también por qué los medios de auxilio tuvieron problemas para intervenir rápidamente. El espacio del centro concentraba en pleno día un tráfico vehicular denso para responder a las necesidades de movilidad de los habitantes, que iban al centro de la aglomeración a hacer sus compras preparativas del carnaval 2002. Con la escorrentía pluvial sobre las calzadas, los choferes perdieron el control de los vehículos que obstruyeron calles estrechas con pendientes abruptas, obstaculizando considerablemente el desplazamiento de los vehículos de auxilio en este espacio del centro.

El establecimiento de las temporalidades de la crisis invita, en un segundo tiempo, a identificar los espacios de la crisis, para luego establecer los vínculos existentes. Se puede distinguir al menos dos tipos de espacios: los espacios donde la crisis tuvo lugar, es decir los espacios afectados, y los espacios donde los elementos de gestión de crisis eran movilizables a favor de los espacios afectados. Enfocándose únicamente en los elementos de gestión de crisis, los centros hospitalarios requeridos están localizados en el espacio que ocupa el barrio Miraflores. Los espacios afectados son el centro de la ciudad y los barrios de la parte sur edificados en las inmediaciones del torrente Choqueyapu. Para comprender cómo los elementos de auxilio médicos han podido ir en auxilio a los espacios afectados, hay que observar la accesibilidad de estos dos espacios. Ahora bien, el 19 de febrero de 2002, las inundaciones interrumpieron la movilidad en vías de comunicación

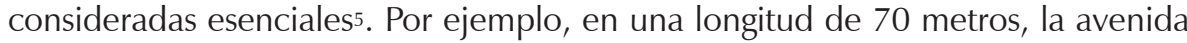
que unía el barrio La Florida al barrio Aranjuez, en la parte sur de la ciudad, fue destruida, aislando Aranjuez del resto de la ciudad, principalmente de Miraflores. Por su lado, la concentración del aguacero de granizo sobre el centro provocó la pérdida de ciertos tramos de vías de comunicación y, por lo tanto, de la movilidad sobre esta parte central de la red vial, la cual provocó también la pérdida de accesibilidad a otros espacios de la ciudad, como Miraflores, a pesar de ser esenciales para una rápida resolución de la situación. Por ejemplo, fueron difíciles el acceso y la salida de los medios de auxilio como las ambulancias, entre la zona de impacto del evento en el centro y el barrio Miraflores. En período de urgencia, la rapidez de intervención de los auxilios es primordial, sobre todo cuando los heridos sufren de hipotermia. Pero esta rapidez fue afectada por la pérdida de

5 Ver en este volumen, el artículo de Núñez \& Demoraes, pp. 827-848. 
accesibilidad, por lo tanto tuvo consecuencias negativas sobre la mortalidad. Solo a partir de las 17:00 los tres establecimientos de salud requeridos en el barrio de Miraflores (Hospital de Clínicas, Hospital del Tórax, Asistencia Pública) pudieron comenzar a recibir a los primeros heridos (Villegas, 2002: 19).

\section{INCIDENCIAS}

El reconocimiento del evento como un desastre llevó a las autoridades municipales y nacionales a tomar decisiones en materia de gestión de los riesgos. Desde este punto de vista, la crisis se convirtió en un modelo, al menos para el GMLP, que sirve todavía de patrón. Las respuestas del GMLP para intentar administrar los riesgos se estructuraron alrededor de tres enfoques: la planificación, la prevención y la preparación a los riesgos. Por ejemplo, en términos de planificación, el GMLP, con ayuda de una financiación canalizada por el PNUD, lanzó, desde el segundo semestre de 2002, un estudio de los riesgos a nivel municipal, que ha desembocado en una serie de mapas de riesgos (Ayala et al., 2004), los cuales orientan hoy las políticas municipales de gestión de los riesgos (GMLP, 2007). Por ejemplo, los habitantes de las zonas de alto riesgo, identificados por estos mapas, se benefician por parte del GMLP de una exoneración parcial de impuestos, en reconocimiento de la exposición de sus bienes a daños. Asimismo, la constatación de las deficiencias funcionales del sistema de drenaje pluvial, en febrero de 2002, que no había sido revisado desde su construcción en 1972, convenció al GMLP de efectuar un diagnóstico detenido del sistema. Este documento le permite mejorar las acciones preventivas, ayudándolo a decidir sobre las reparaciones más urgentes a realizar en el sistema de drenaje de las aguas pluviales, y orientándolo también sobre las mejoras y extensiones necesarias del sistema (Hardy, 2009).

En el ámbito de la preparación, el GMLP ha imaginado una estructura de gestión de las situaciones de urgencia a partir de la organización que surgió espontáneamente durante la crisis. Efectivamente, la ley n. ${ }^{\circ} 2028$ de los municipios del 28 de octubre de 1999 establece que los gobiernos municipales deben ejecutar planes y programas que permitan eliminar o reducir las causas y efectos de las catástrofes naturales, así como aquellas provocadas por el hombre, a través de mecanismos financieros, educativos y técnicos. A pesar de los eventos catastróficos anteriores, el GMLP nunca había tomado plena conciencia de esta obligación, no obstante necesaria para anticipar una crisis. El evento del 19 de febrero de 2002 ha representado una profunda conmoción que le ha hecho recuperar su retraso. En marzo de 2002, la resolución municipal n. ${ }^{\circ}$ 0045/2002 creó un Comité permanente de atención y de gestión de las catástrofes y de las situaciones de urgencia de la Municipalidad de La Paz, que progresivamente se convertirá en el Comité permanente de gestión de los riesgos. Pero la herramienta de gestión de las crisis es sin duda alguna el Comité de operación de emergencia (COE), que fue establecido por la resolución municipal n. ${ }^{\circ}$ 0344/2002 del 29 de noviembre de 2002.

Con los sucesos de la granizada de 2002, los responsables municipales tomaron conciencia que la decisión de subinvertir en la gestión de los riesgos durante los 
años anteriores había tenido consecuencias muy costosas en el plano económico, humano y ciertamente en el plano político... ya que los ciudadanos criticaron severamente la gestión del evento por parte de las autoridades municipales. Consecuentemente, a partir de 2002, las decisiones municipales vinculadas a los riesgos estuvieron acompañadas de un apoyo financiero importante (fig. 2). Durante la primera mitad de los años 1990, la inversión destinada a la gestión de los riesgos había disminuido considerablemente, incluso hasta no representar nada entre 1992 y 1994. Entre 1995 y 2002, si aumentó sólo fue para recuperar la mitad de las sumas invertidas a fines de los años 1980. Entre 2002 y 2004, los montos invertidos fueron nuevamente muy importantes (hasta más de 10 millones de dólares en 2004), para volver progresivamente a los elevados niveles de las inversiones de los años 1980, es decir entre 5 y 6 millones de dólares por año. Estas sumas estaban destinadas tanto a las políticas de prevención, que consistían principalmente en construcciones de obras de ingeniería hidráulica (tubos de desagüe, canales, etc.), como a la preparación para la crisis (capacitación del personal del COE, compra de material de primera necesidad, etc.) y a la planificación (realización de un mapa de los riesgos, realización de un mapa de los derechos de servidumbre alrededor de los cursos de agua, etc.).

A nivel nacional, también se aprendieron lecciones de la catástrofe del 19 de febrero de 2002. La ley n. 2140 del 25 de octubre de 2000 distribuía los roles entre los actores institucionales en materia de reducción de los riesgos y de gestión de las crisis, obligando sobre todo las municipalidades a prepararse.

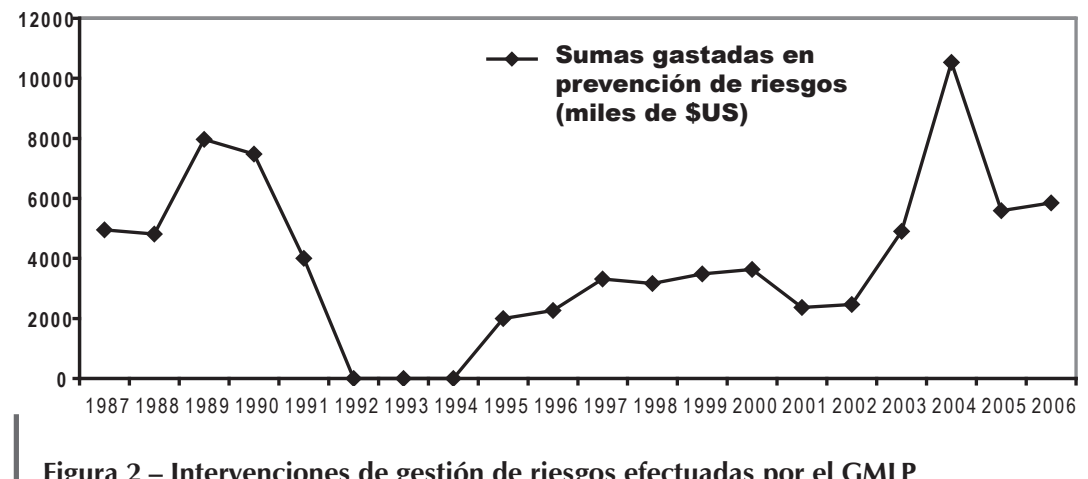

Figura 2 - Intervenciones de gestión de riesgos efectuadas por el GMLP Fuente: Dossier estadístico del Municipio de La Paz 2000-2005

Sin embargo, la ley no preveía verdaderamente una transferencia de medios para ayudar las municipalidades a cumplir con esta obligación. El 5 de marzo de 2002, esta ley fue modificada por la ley . $^{\circ} 2335$ que pretendía remediar algunos de los problemas que surgieron con versión del evento del 19 de febrero 2002. Y fue nuevamente modificada después de los movimientos sociales de febrero de 2003 por la ley n. ${ }^{\circ} 2442$. 
Por otro lado, los organismos internacionales también han contribuido a mejorar la preparación ante la crisis. Por ejemplo, la Organización Panamericana de la Salud (OPS) se dedicó a reforzar la Unidad de Preparación y de Respuesta a los Desastres (Upred) del ministerio de Salud, a partir de capacitaciones dirigidas a sus responsables en los campos de las operaciones y de la logística (identificación de almacenes estratégicos de medicamentos), de la preparación, y de la vigilancia de las epidemias después de un desastre (OPS, 2002).

Se observa así que las políticas de gestión de las crisis, a nivel nacional y sobre todo municipal, han sido modificadas considerablemente a partir del evento de febrero de 2002, que se ha convertido en el modelo patrón de la crisis catastrófica de origen natural. Sin embargo, las mejoras aportadas no prescinden de interrogar su eficacia. Estas consisten en gran medida en la prevención esperada por parte de obras de ingeniería civil costosas que permitirían enfrentar las amenazas. Si bien desde 2002 La Paz no ha sufrido crisis de origen natural, en cambio ha sufrido por lo menos dos crisis de importantes consecuencias: la crisis de origen social de octubre de 2003 y la crisis vinculada a la ruptura de abastecimiento de agua potable en enero-febrero de 20086 ${ }^{6}$. Esto tiende a demostrar que el enfoque preventivo limitado a las amenazas no reduce las vulnerabilidades profundas de la ciudad. Eso por las dificultades de los gestores de crisis en apreciar los espacios del funcionamiento urbano vulnerables, los vínculos entre los espacios vulnerables y los espacios de gestión de las crisis.

\section{Referencias citadas}

AYALA, R., MALDONADO, H., GANDARILLAS, C. \& HUANCA, T. M., 2004 - Mapa de riesgo socio natural específico de la ciudad de La Paz: memoria explicativa, 59 pp.; La Paz: OM Técnica-GMLP.

BABY, V., 1995 - El Alto de La Paz. Un bidonville d'altitude au cœur de l'Amérique latine, 142 pp. Mémoire de DEA, Université Paris X, Nanterre.

BCEOM, BRGM, PCA, 1977 - Plan de desarrollo urbano-ciudad de La Paz, 41 tomos; La Paz: HAM.

COMBAZ, E., 2007 - Le relogement des populations sinistrées de la municipalité de La Paz. Contextes, évolutions, et impacts des pratiques de relogement de trois catastrophes : la granizada (2002), Llojeta (2003) et German Jordan (1997), 107 pp. Mémoire de Master 1 de Géographie, mention Sciences géographiques, spécialité Mondialisation et développement, Université de Aix-Marseille 1.

DEMORAES, F., 1998 - Étude de l'évolution de l'agglomération de La Paz-El Alto depuis les vingt dernières années, compte tenu des contraintes environnementales du site, 120 pp.; Chambéry: Université de Savoie.

D'ERCOLE, R. \& METZGER, P., 2004 - Vulnerabilidad del Distrito Metropolitano de Quito, 496 pp.; Quito, Ecuador: MDMQ-IRD. Colección Quito Metropolitano. 
GMLP, 2007 - Catálogo de instrumentos en gestión municipal para la reducción de riesgos y preparación ante emergencias, 74 pp.; La Paz: PNUD.

GMLP, 200-2005 - Dossier estadístico del Municipio de La Paz 2000-2005; La Paz: GMLP.

HARDY, S., 2009 - Explorer la construction de la résilience. Expériences de recherche à La Paz. In: Vulnérabilités sociétales, risques et environnement (A. Pelier \& S. Becerra, eds.): 469-482; París: Éditions L'Harmattan.

LAGADEC, P., 2002 - Crisis Management in France: Trends, Shifts and Perspectives. Journal of Contingencies and Crisis Management, vol. 10, n. ${ }^{\circ} \mathbf{4}$ : 159-172.

NATHAN, F., 2008 - Risk Perception, Risk Management and Vulnerability to Landslides in the hill slopes in the city of La Paz, Bolivia. A Preliminary Statement. Disasters, 32 (3): 337-357.

OPS, 2002 - URL :<http://www.ops.org.bo/ambiental/desastres/inundacion/20020200/>

VILLEGAS, S. (ed.), 2002 - Memoria del «Martes Negro». iEmergencia por granizada en La Paz!, 59 pp.; La Paz: GMLP, Comisión episcopal de pastoral social CARITAS. 